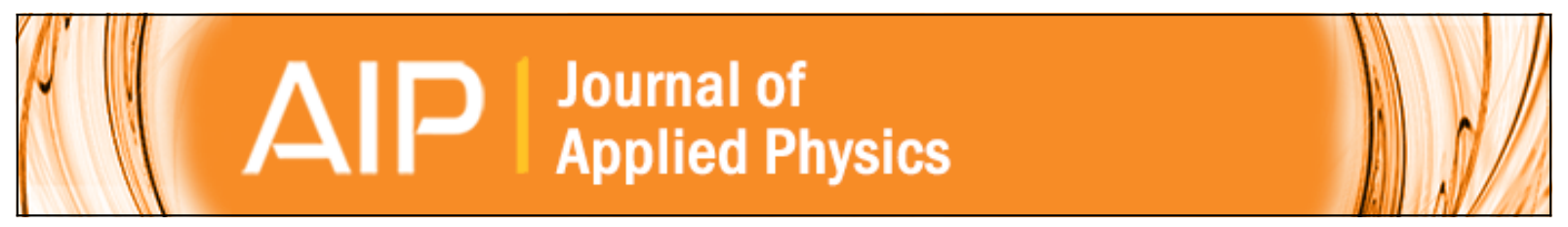

\title{
Soft magnetic properties of high-temperature nanocrystalline alloys: Permeability and magnetoimpedance
}

\author{
J. S. Blázquez, V. Franco, A. Conde, and L. F. Kiss
}

Citation: Journal of Applied Physics 93, 2172 (2003); doi: 10.1063/1.1539294

View online: http://dx.doi.org/10.1063/1.1539294

View Table of Contents: http://scitation.aip.org/content/aip/journal/jap/93/4?ver=pdfcov

Published by the AIP Publishing

\section{Articles you may be interested in}

Effect of magnetic field annealing methods on soft magnetic properties for nanocrystalline

(Fe0.5Co0.5)73.5Si13.5B9Nb3Cu1 alloy

J. Appl. Phys. 117, 17 B729 (2015); 10.1063/1.4917324

Investigation on high-temperature magnetic permeability of Si-rich nanocrystalline

(Fe0.9Co0.1)74.5Nb2Si17.5B5Cu1 alloy

J. Appl. Phys. 117, 17B701 (2015); 10.1063/1.4906297

Magnetic permeability of Si-rich (FeCoNi)-based nanocrystalline alloy: Thermal stability in a wide temperature range

J. Appl. Phys. 113, 17A310 (2013); 10.1063/1.4794718

Effect of $\mathrm{P}$ addition on nanocrystallization and high temperature magnetic properties of low $\mathrm{B}$ and $\mathrm{Nb}$ containing FeCo nanocomposites

J. Appl. Phys. 111, 07A301 (2012); 10.1063/1.3670056

High-temperature giant magnetoimpedance in Fe-based nanocrystalline alloy

J. Appl. Phys. 87, 5263 (2000); 10.1063/1.373315

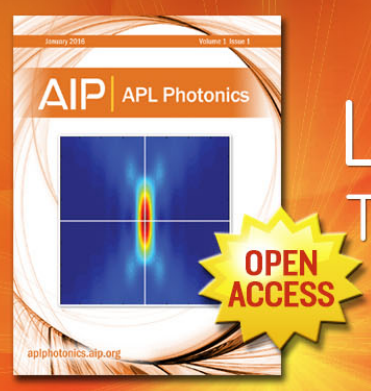

Launching in 2016!

The future of applied photonics research is here 


\title{
Soft magnetic properties of high-temperature nanocrystalline alloys: Permeability and magnetoimpedance
}

\author{
J. S. Blázquez, V. Franco, and A. Conde ${ }^{a}$ \\ Departamento de Física de la Materia Condensada, ICMSE-CSIC, Universidad de Sevilla, P. O. Box 1065, \\ 41080 Sevilla, Spain \\ L. F. Kiss \\ Research Institute for Solid State Physics and Optics, Hungarian Academy of Sciences, P. O. Box 49, 1525 \\ Budapest, Hungary
}

(Received 5 June 2002; accepted 21 November 2002)

\begin{abstract}
The technological applicability of $\mathrm{FeCoNbBCu}$ alloys is suggested in terms of measurements of room temperature magnetoimpedance and temperature dependence of magnetic permeability $\mu_{r}$. Results for the $\mathrm{Fe}_{78-x} \mathrm{Co}_{x} \mathrm{Nb}_{6} \mathrm{~B}_{15} \mathrm{Cu}_{1}$ alloy series show that room temperature soft magnetic properties are enhanced in the lowest Co containing alloy ( $\mu_{r} \sim 10500$ and magnetoimpedance ratio $\sim 60 \%$ at $1 \mathrm{MHz}$ ). However, permeability exhibits a smoother thermal dependence in the alloys with medium and high Co content. A tradeoff between magnetic softness and its thermal stability reveals the alloy with 39 at. \% Co as the most suitable composition among those studied, characterized by a temperature coefficient of $\sim 0.02 \% / \mathrm{K}$ from room temperature up to $900 \mathrm{~K}$. This value is 1 order of magnitude smaller than those observed for FeSiBCuNb (FINEMET-type) alloys and Mn ferrites and extended over a much wider temperature range than in these materials. (C) 2003 American Institute of Physics. [DOI: 10.1063/1.1539294]
\end{abstract}

\section{INTRODUCTION}

Soft magnetic nanocrystalline alloys have been profusely studied since the development of FINEMET $(\mathrm{FeSiBNbCu})^{1}$ and NANOPERM alloys (FeMB and $\mathrm{FeMBCu}, \mathrm{M}=\mathrm{Nb}, \mathrm{Zr}$, Hf...). ${ }^{2}$ These materials present a two-phase microstructure, in which ferromagnetic nanocrystals ( $\alpha$-FeSi or $\alpha$-Fe, respectively) are embedded in a residual ferromagnetic amorphous phase. Their outstanding soft-magnetic properties at room temperature are widely covered in the literature. ${ }^{3}$ However, as the temperature increases, soft magnetic properties are lost due to the transition of the amorphous phase to the paramagnetic state, ${ }^{4}$ which limits the applicability of these materials at high temperatures. Searching for soft magnetic nanocrystalline alloys with high temperature applicability led to the development of HITPERM-type alloys $(\mathrm{FeCoMBCu}),{ }^{5}$ in which partial substitution of $\mathrm{Fe}$ by $\mathrm{Co}$ was shown to enhance the Curie temperature of the amorphous phase.

The higher coercivity exhibited by HITPERM alloys, compared to FINEMET and NANOPERM, has been explained in terms of the different evolution of the saturation magnetostriction constant $\lambda_{S}$ during nanocrystallization. Whereas in FINEMET and NANOPERM alloys the contributions to $\lambda_{S}$ coming from the crystalline and residual amorphous phases are of different sign, giving an overall value close to zero, in HITPERM alloys both contributions are positive. ${ }^{3}$ In this case, the softness of the material is controlled by the relative variation of the magnetostriction from the amorphous phase to the crystalline one. ${ }^{6}$

a) Author to whom correspondence should be addressed; electronic mail: conde@us.es
There have been numerous studies concerning the microstructural characterization and magnetic phase transition in HITPERM-type alloys. ${ }^{3,5,7-10}$ However, there are still few results on magnetic properties relevant for possible applications of these materials, such as magnetic permeability $\mu_{r}$ and giant magnetoimpedance effect (GMI). The latter has been a field of intensive research in recent years; ${ }^{11-13}$ to date it is a new way for the application of soft magnetic materials. ${ }^{14-17}$ There are numerous studies of the different factors affecting the magnetoimpedance behavior of soft magnetic nanocrystalline alloys as FINEMET and NANOPERM, analyzing, for example, the influence of the thermal treatments, ${ }^{18-25}$ compositional effects, ${ }^{18-20}$ the influence of domain structure and induced anisotropies, ${ }^{21-23}$ the effect of the measuring frequency, ${ }^{19-22,24}$ and temperature, ${ }^{24,25}$ etc. However, there is a lack of data in the literature concerning the HITPERM family of alloys.

In this work the influence of the Co to Fe ratio on the room temperature magnetoimpedance and thermal dependence of magnetic permeability of HITPERM-type alloys has been studied. It will be shown that although Co addition deteriorates the room temperature soft magnetic properties of the alloy, a wider temperature range of stable magnetic properties is obtained.

\section{EXPERIMENT}

Amorphous ribbons, $\sim 20 \mu \mathrm{m}$ thick and $\sim 5 \mathrm{~mm}$ wide, of nominal compositions $\mathrm{Fe}_{78-x} \mathrm{Co}_{x} \mathrm{Nb}_{6} \mathrm{~B}_{15} \mathrm{Cu}_{1}(x=18,39$, 60) were produced at Warsaw University of Technology by the single roller melt-spinning technique. The devitrification 
TABLE I. Thermal treatments of samples submitted to magnetoimpedance experiments. Initial nanocrystalline corresponds to samples with low crystalline volume fraction, while final nanocrystalline corresponds to samples annealed up to the end of the first crystallization stage.

\begin{tabular}{lcc}
\hline \hline Alloy & Sample & Heat treatment \\
\hline & Structurally relaxed & $15 \mathrm{~min}$ at $600 \mathrm{~K}$ \\
$\mathrm{Fe}_{60} \mathrm{Co}_{18} \mathrm{Nb}_{6} \mathrm{~B}_{15} \mathrm{Cu}_{1}$ & Initial nanocrystalline & $15 \mathrm{~min}$ at $725 \mathrm{~K}$ \\
& Final nanocrystalline & $15 \mathrm{~min}$ at $800 \mathrm{~K}$ \\
& Structurally relaxed & 15 min at $600 \mathrm{~K}$ \\
& Initial nanocrystalline & 15 min at $725 \mathrm{~K}$ \\
$\mathrm{Fe}_{39} \mathrm{Co}_{39} \mathrm{Nb}_{6} \mathrm{~B}_{15} \mathrm{Cu}_{1}$ & Final nanocrystalline & $15 \mathrm{~min}$ at $850 \mathrm{~K}$ \\
& Structurally relaxed & $15 \mathrm{~min}$ at $600 \mathrm{~K}$ \\
& Initial nanocrystalline & 15 min at $700 \mathrm{~K}$ \\
$\mathrm{Fe}_{18} \mathrm{Co}_{60} \mathrm{Nb}_{6} \mathrm{~B}_{15} \mathrm{Cu}_{1}$ & Final nanocrystalline & $15 \mathrm{~min}$ at $800 \mathrm{~K}$ \\
& &
\end{tabular}

process of the alloys has been studied by differential scanning calorimetry (DSC), transmission electron microscopy and $\mathrm{x}$-ray diffraction techniques.

Permeability measurements were performed on toroidal samples at a frequency of $6 \mathrm{kHz}$ and an applied field low enough to assure the measurement of initial permeability $(\sim$ $0.5 \mathrm{~A} / \mathrm{m})$. The experiment consisted of several heatingcooling cycles and both the measurement and heat treatment of the samples were performed at the same time. For these measurements two coils were wounded around the sample. With the first coil an ac field is applied to the sample, whereas the signal induced in the second coil is detected using a lock-in technique. An impedance analyzer (HewlettPackard 4192A) was used to calibrate the values of permeability at room temperature.

For magnetoimpedance measurements the impedance analyzer was used. Two electrical contacts were spot welded to the samples at a distance of $5 \mathrm{~cm}$. DSC experiments confirmed that this procedure did not affect the devitrification process of the alloys studied. Samples were annealed in a halogen-lamp furnace in vacuum at different stages of the devitrification process presented in Table I. For measuring the field dependence of the impedance, an ac intensity of 10 $\mathrm{mA}$ at frequencies ranging from 0.5 to $5 \mathrm{MHz}$ was applied through the sample. The maximum applied field was 7.2 $\mathrm{kA} / \mathrm{m}$.

\section{RESULTS}

\section{A. Devitrification process}

The devitrification process of the as-cast samples occurs in two main stages. ${ }^{26}$ During the first one, a nanocrystalline microstructure is formed, in which $\alpha$-FeCo nanocrystals with an average grain size of $\sim 5 \mathrm{~nm}$ are embedded in a residual amorphous matrix. The crystalline volume fraction at the end of the nanocrystallization process is $\sim 55 \%$ for the alloys with 18 and 39 at. \% Co, but it is lower in the alloy with 60 at. \% Co $(\sim 45 \%) .{ }^{26}$ At higher temperatures $(>900 \mathrm{~K})$, recrystallization of a fraction of the $\alpha$-FeCo crystallites and the residual amorphous matrix produces a fully crystalline alloy, with the appearance of boride phases, mainly (FeCo) $(\mathrm{FeCo})_{23} \mathrm{~B}_{6} \cdot{ }^{27}$

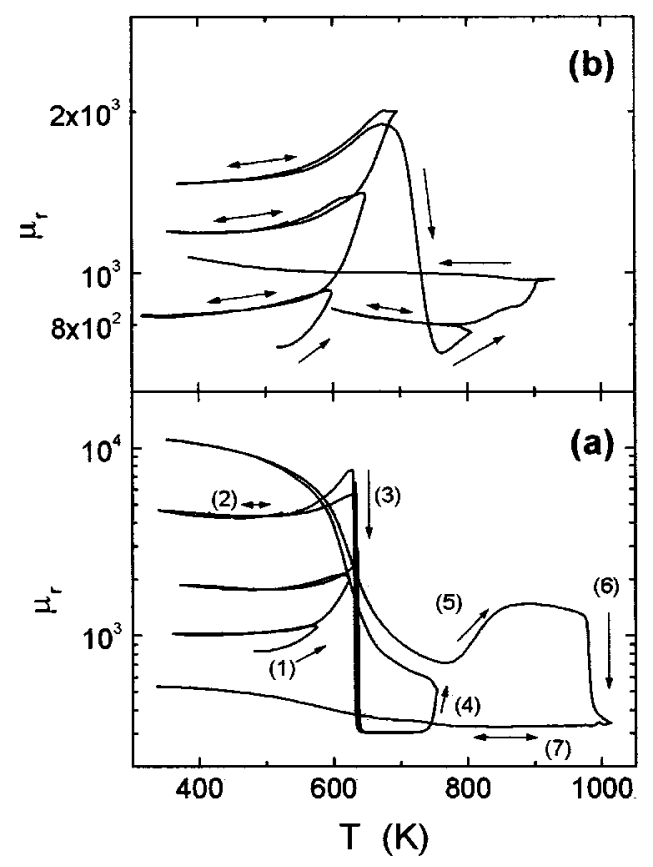

FIG. 1. Initial permeability vs temperature in successive heating-cooling cycles for: (a) $\mathrm{Fe}_{60} \mathrm{Co}_{18} \mathrm{Nb}_{6} \mathrm{~B}_{15} \mathrm{Cu}_{1}$ and (b) $\mathrm{Fe}_{39} \mathrm{Co}_{39} \mathrm{Nb}_{6} \mathrm{~B}_{15} \mathrm{Cu}_{1}$ alloys. Numbers indicate the sequence of heating-cooling cycles.

\section{B. Initial permeability}

Figure 1(a) shows the evolution of the initial permeability with temperature for $\mathrm{Fe}_{60} \mathrm{Co}_{18} \mathrm{Nb}_{6} \mathrm{~B}_{15} \mathrm{Cu}_{1}$ as-cast sample submitted to continuous heating and cooling cycles. The characteristic features have been numbered in the figure. Initially (1) the relaxation of the internal stresses provokes a progressive increase of the room temperature permeability; cooling cycles (2) show that permeability remains constant for temperatures lower than $550 \mathrm{~K}$ at this stage. A characteristic Hopkinson peak is observed at the Curie temperature of the amorphous phase, which is due to a faster decrease of magnetic anisotropy than that of magnetization with increasing temperature, and thus the permeability diverges at the Curie point. ${ }^{28}$ Above the Curie point of the amorphous phase, an abrupt fall in the permeability is observed (3), due to the transition of the system to the paramagnetic state. At higher temperatures, an increase of permeability at the onset of nanocrystallization occurs (4), which is connected with the appearance of the ferromagnetic nanocrystals of the $\alpha$-FeCo phase. ${ }^{29}$ When this microstructure is achieved, the system shows the highest room temperature permeability. However, no Hopkinson peak can be detected at the Curie point of the residual amorphous phase $\left(T_{C}^{\mathrm{am}}\right)$, which can be due to the non-zero value of the effective magnetic anisotropy of the whole system: the nanocrystals present a much higher Curie temperature than the amorphous matrix and therefore, $\mu_{r}$ does not diverge at $T_{C}^{\mathrm{am}}$. Also, the fall in $\mu_{r}$ at $T_{C}^{\mathrm{am}}$ is smoother for the nanocrystallized sample than for the fully amorphous one. In fact, the residual amorphous matrix is not homogeneous ${ }^{30}$ and thus the Curie temperature is not well defined. ${ }^{31}$ For this alloy, room temperature permeability shows high values at this stage, but it depends strongly on temperature, which is a nondesirable effect for high tempera- 


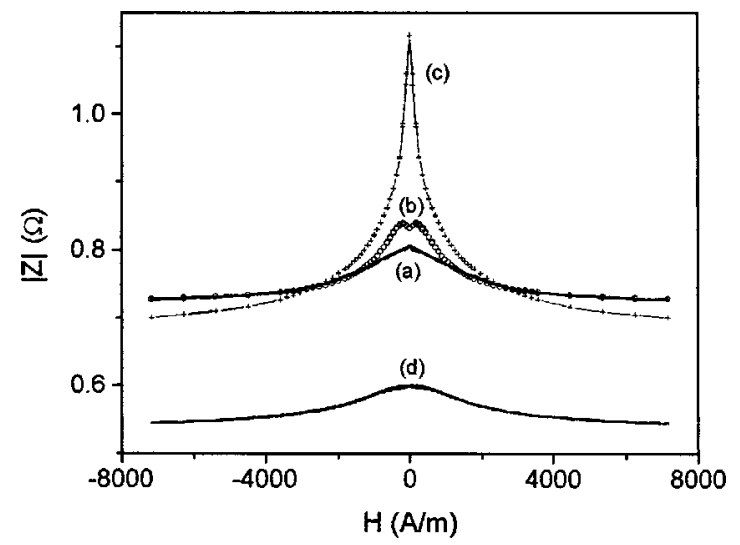

FIG. 2. Magnetoimpedance modulus of $\mathrm{Fe}_{60} \mathrm{Co}_{18} \mathrm{Nb}_{6} \mathrm{~B}_{15} \mathrm{Cu}_{1}$ alloy: (a) ascast, (b) structurally relaxed, (c) initial-nanocrystalline, and (d) finalnanocrystalline samples.

ture applications of the material. As nanocrystallization progresses, permeability increases (5), but for temperatures above the second transformation stage permeability decreases (6), and the soft magnetic properties of the material are lost in all the explored temperature range (7) due to the presence of boride-type phases. The slight increase in $\mu_{r}$ as temperature decreases can be explained in terms of the Curie temperatures of the boride-type phases formed, especially the $(\mathrm{FeCo})_{23} \mathrm{~B}_{6}$ phase, with $T_{C}=784 \mathrm{~K}^{32}$

The behavior of the other alloys studied shows similar trends, however a wider temperature range of constant permeability can be observed for the nanocrystalline samples of the alloys with higher Co content [Fig. 1(b)]: In contrast with the 18 at. \% Co alloy, where $\mu_{r}$ decreases at temperatures above $500 \mathrm{~K}$, the alloys with $39 \%$ and $60 \%$ have a constant value of permeability up to 950 and $900 \mathrm{~K}$, respectively. This can be ascribed to the higher Curie temperature of the amorphous matrix, which might allow the magnetic coupling between the nanocrystals through the matrix up to higher temperatures. ${ }^{4}$

\section{Magnetoimpedance}

Figure 2 shows the modulus of impedance $(|Z|)$ versus dc applied magnetic field $(H)$, obtained at room temperature at a frequency of $1 \mathrm{MHz}$, for samples of the 18 at. \% Co alloy in the as-quenched state and after annealing. The value of $|Z|$ at the maximum applied field decreases as the crystalline fraction increases, being the same for as-cast and relaxed samples, in agreement with the reduction of dc resistivity with the progress of crystallization.

Usually the GMI effect is described in terms of the magnetoimpedance ratio (MIR ), calculated as:

$$
\operatorname{MIR}=100 \cdot \frac{|Z(H)|-\left|Z\left(H_{\max }\right)\right|}{\left|Z\left(H_{\max }\right)\right|},
$$

where $\left|Z\left(H_{\max }\right)\right|$ is the impedance modulus measured at maximum applied field $(7200 \mathrm{~A} / \mathrm{m})$. Figure 3 shows MIR values versus $H$, for all the studied alloys in the as-cast, structurally relaxed, initial-nanocrystalline and finalnanocrystalline states. The maximum value of MIR versus annealing temperature is represented in Fig. 4. For the alloy

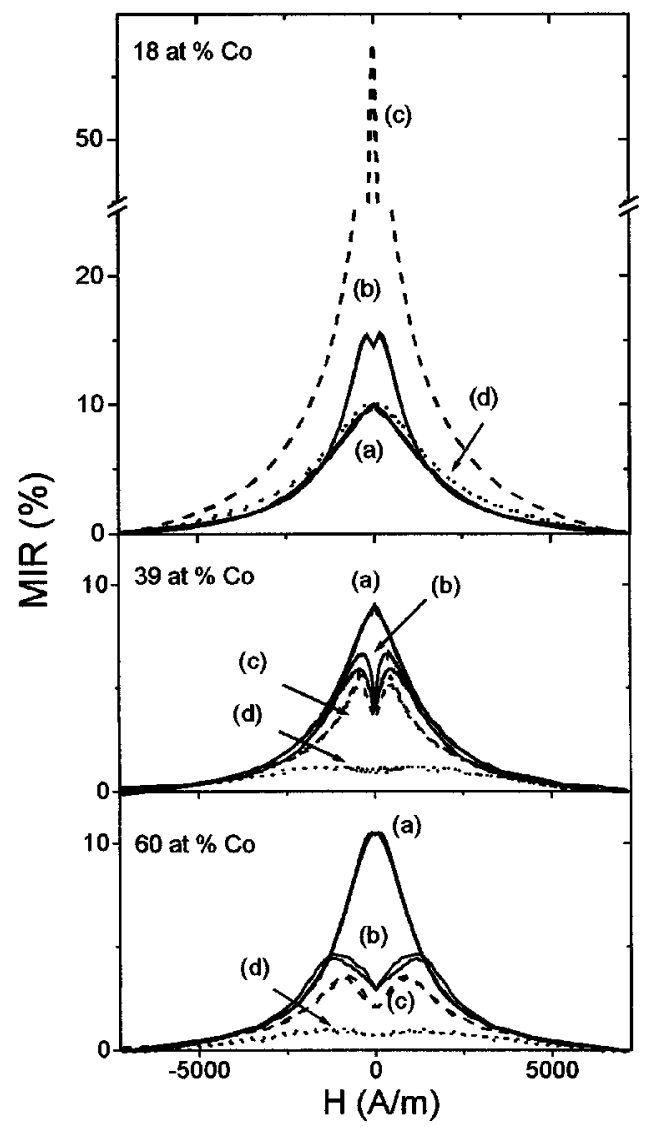

FIG. 3. Magnetoimpedance ratio for the different alloys studied: (a) as-cast, (b) structurally-relaxed, (c) initial-nanocrystalline, and (d) finalnanocrystalline samples

with 18 at. \% Co the GMI effect increases for the relaxed sample with respect to the as-cast sample, reaches a maximum value for the initial-nanocrystalline sample $(\sim 60 \%)$ and decreases for the final-nanocrystalline sample. However, for the higher Co content alloys, the maximum GMI effect appears for the as-cast samples, decreasing as the annealing temperature increases.

The magnetoimpedance scans (Fig. 3) show a singlepeak structure for all the as-cast samples studied and for the initial-nanocrystalline sample of the 18 at. \% Co alloy; however a double peak structure can be observed for all the other

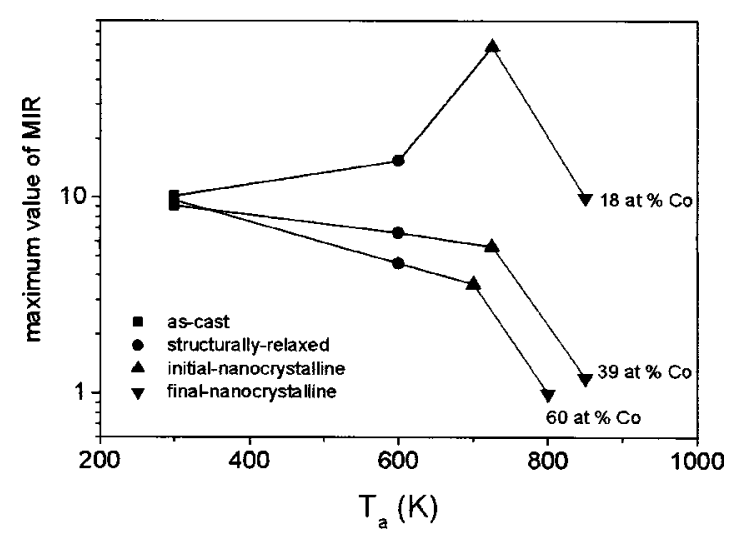

FIG. 4. Influence of the microstructure on the maximum magnetoimpedance ratio. 


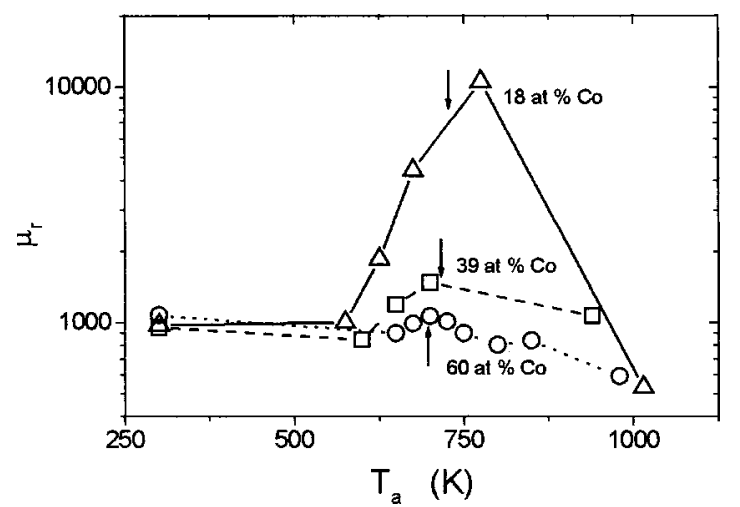

FIG. 5. Initial permeability measured at $400 \mathrm{~K}$ vs maximum reached temperature in the previous treatment. Arrows indicate the crystallization processes detected by DSC.

samples studied. This kind of two-peak structure and the hysteretic behavior of magnetoimpedance are deleterious for the applicability of the alloys.

\section{DISCUSSION}

The behavior of the permeability and magnetoimpedance is correlated with the previously reported behavior for coercivity and magnetostriction. ${ }^{6,26}$ As the factors that affect the magnetic properties depend strongly on the microstructure, it is suitable to divide the samples studied in amorphous samples (as-cast and structurally relaxed samples) and nanocrystalline samples (annealed at the beginning and at the end of the nanocrystallization).

\section{A. Amorphous samples}

Figure 5 shows the initial permeability measured at 400 $\mathrm{K}$ (maximum temperature below which $\mu_{r}$ remains approximately constant for all the alloys studied and not producing further structural transformation of the samples) versus maximum achieved temperature in the previous heat treatment of the continuous heating-cooling cycle experiments. In this figure it is possible to observe the evolution of $\mu_{r}$ with the microstructure. An increase in permeability with the structural relaxation phenomena can be found for all the studied alloys, being the effect larger as the Co content decreases in the alloy. This evolution differs from the one reported for coercivity ${ }^{26}$ and from the observed evolution in MIR (Fig. 4). In fact, only for the 18 at. \% Co alloy is a clear softening of the magnetic properties observed after structural relaxation, reflected in the magnetoimpedance results as an increase in the GMI effect in structurally relaxed samples.

This difference can be understood in terms of the previously proposed mechanism for the increase in room temperature coercivity: the domain wall stabilization phenomena. ${ }^{6}$ Permeability measurements and heat treatments of the samples were made simultaneously, thus the annealing was under the presence of an ac field. It has been reported that annealing under ac field diminishes (or even nulls) the domain wall stabilization effect, ${ }^{33}$ which can explain the different evolution of the permeability and the coercivity with the structural relaxation.
As it has been pointed before, the behavior of MIR with the structural relaxation agrees with the behavior found for the coercivity. ${ }^{6,26}$ However, the domain wall stabilization phenomena would be a suitable explanation for the magnetic hardening of the higher Co content alloys only if it is possible to confirm that the domain wall motion is a contributing mechanism to the magnetization process at the frequency used in the magnetoimpedance experiments (1 MHz). The frequency evolution of MIR in nanocrystalline alloys shows that the maximum value of MIR increases up to a certain value of frequency, after which it decreases. This fall in the GMI effect has been associated with the disappearance of the contribution of the domain wall motion to the magnetization process at high frequencies. ${ }^{15,34}$ Therefore, the measuring frequency was changed from 0.5 to $5 \mathrm{MHz}$ in the magnetoimpedance experiments. A continuous increase in MIR was observed as the applied frequency is increased, confirming that the domain wall motion is a contributing mechanism to the magnetization process at $1 \mathrm{MHz}$ and, therefore, the domain wall stabilization is a suitable explanation for the magnetic hardening of the high Co content alloys.

The two-peak structure observed in the magnetoimpedance scans is related to the magnetic anisotropy field, $H_{K}$, being equal to the observed shift of the maximum value of MIR with respect to $H=0 .{ }^{34}$ In our case, this is true for the 18 at. \% Co containing alloy, however, because the domain wall stabilization phenomena affect the position of the maximum value of MIR, ${ }^{35}$ it is not possible to affirm the same for the 39 and 60 at. \% Co alloys.

In amorphous samples, the main contribution to the anisotropy field is the magnetoelastic anisotropy, and $H_{K}$ can be expressed as

$$
H_{K}=\frac{2 K}{\mu_{0} M_{S}}=\frac{3 \lambda_{S} \sigma}{\mu_{0} M_{S}},
$$

where $K=(3 / 2) \lambda_{S} \sigma$, is the magnetoelastic anisotropy constant, $\lambda_{S}$ the saturation magnetostriction constant, $\sigma$ the internal stresses, $\mu_{0}$ the vacuum permeability, and $M_{S}$ the saturation magnetization. For the structurally relaxed sample of the 18 at. \% Co alloy $H_{K}$, obtained from the position of maximum value of MIR, is $\sim 200 \mathrm{~A} / \mathrm{m}$. The values of $\lambda_{S}$ and $\mu_{0} M_{S}$ for this sample are $30 \mathrm{ppm}$ and $1.1 \mathrm{~T}$, respectively, which gives a value of $\sim 1 \mathrm{MPa}$ for the internal stresses and an anisotropy constant value comparable to those found in other metallic glasses with similar saturation magnetostriction constants. ${ }^{36}$

\section{B. Nanocrystalline samples}

Both the permeability and magnetoimpedance results agree with the reported behavior of coercivity after the nanocrystallization process, ${ }^{26}$ in which the coercivity decreases at the beginning of the nanocrystallization only for the alloy with 18 at. \% Co. For this alloy, the initial permeability shows high values at low temperatures $(<500 \mathrm{~K})$ in nanocrystallized samples $(\sim 10500)$ but low values can be found in the other alloys $(\sim 1000)$. The very low values obtained for samples annealed after the second transforma- 
tion stages are due to the formation of boride-type phases which drastically harden the magnetic properties.

For other HITPERM-type composition, $\mathrm{Fe}_{44} \mathrm{Co}_{447}$ $\mathrm{B}_{4} \mathrm{Cu}_{1},{ }^{8}$ the maximum initial permeability measured at room temperature was 1800 , obtained at $0.4 \mathrm{kHz}$. At $6 \mathrm{kHz}$ (measuring frequency in our experiments) the value of $\mu_{r}$ is $\sim 1000$, similar to the obtained value for our nanocrystallized alloy $\mathrm{Fe}_{39} \mathrm{Co}_{39} \mathrm{Nb}_{6} \mathrm{~B}_{15} \mathrm{Cu}_{1}$, but 1 order of magnitude lower than the maximum value exhibited by the nanocrystalline samples of $\mathrm{Fe}_{60} \mathrm{Co}_{18} \mathrm{Nb}_{6} \mathrm{~B}_{15} \mathrm{Cu}_{1}$ ( $\left.\sim 10500\right)$.

The evolution of permeability and the decrease of resistivity with nanocrystallization must affect the evolution of the GMI effect. ${ }^{37}$ For samples of the different alloys at the same annealing stage (structurally relaxed, initial nanocrystalline and final nanocrystalline), the $|Z|$ values at the maximum applied field are similar, indicating that resistivity does not differ considerably between these samples. Therefore, the compositional effect on GMI for the series studied might be ascribed to the different values of permeability.

The GMI effect increases at the beginning of the nanocrystallization only for the alloy with 18 at. \% Co, as it has been previously observed for FINEMET and NANOPERM compositions. ${ }^{37}$ For these alloys, both the enhancement of magnetic softness and the decrease of resistivity contribute to the increase in the GMI effect. However, for the 39 and 60 at. \% Co alloys the GMI effect is lower in the nanocrystallized samples than in the amorphous samples, despite the decrease of the resistivity but in agreement with the observed behavior of the initial permeability and the reported evolution of the coercivity. ${ }^{26}$ This kind of correlation between the evolution of resistivity and GMI effect has also been observed for other nanocrystalline alloys, where a decrease of resistivity at advanced stages of nanocrystallization cannot avoid the decrease of the GMI effect. ${ }^{37}$

The different evolution of the magnetostriction constant of the different alloys can explain the different observed behavior. In fact, the nanocrystalline $\alpha$-FeCo phase that appears in the 39 and 60 at. \% Co alloys with compositions close to $\mathrm{Fe}_{61} \mathrm{Co}_{39}$ and $\mathrm{Fe}_{40} \mathrm{Co}_{60}$, respectively, ${ }^{38}$ shows a higher $\lambda_{S}$ value $(\sim 65 \mathrm{ppm})^{39}$ than that corresponding to the amorphous alloy $(\sim 10-15 \mathrm{ppm})^{6}$ and this makes the net magnetostriction constant increase as the nanocrystallization progresses. However, for the alloy with 18 at. \% Co, the magnetostriction constant is $\sim 15 \mathrm{ppm}$ for both the amorphous ${ }^{6}$ and $\alpha-\mathrm{Fe}_{82} \mathrm{Co}_{18}$ crystalline phases. ${ }^{39}$ For FINEMET and NANOPERM alloys, $\lambda_{S}$ is negative in the nanocrystals and positive in the amorphous phase, producing a decrease of the net magnetostriction of the system and, consequently, an enhancement of the magnetic properties at room temperature. ${ }^{3}$

An important feature for possible applications of these materials is the temperature range of constant permeability. Studies on the temperature dependence of the initial permeability in nanocrystallized FINEMET-type alloys ${ }^{40}$ show that $\mu_{r}$ abruptly decreases at temperatures higher than $575 \mathrm{~K}$. To characterize quantitatively the temperature stability of $\mu_{r}$, the temperature coefficient of permeability can be used as a parameter for comparison. Whereas in FINEMET alloys permeability decreases at a rate of $\sim 0.3 \% / \mathrm{K}$ in a temperature range from 400 to $500 \mathrm{~K},{ }^{40}$ in the $\mathrm{Fe}_{39} \mathrm{Co}_{39} \mathrm{Nb}_{6} \mathrm{~B}_{15} \mathrm{Cu}_{1}$ alloy the temperature coefficient is $\sim 0.02 \% / \mathrm{K}$ in a temperature range from 400 to $900 \mathrm{~K}$, indicating an improvement in the high temperature applications of this alloy with respect to FINEMET. This parameter is very important for some technological applications as $L C$ filters, where ferrites are usually used. A typical value of the temperature coefficient of permeability in Mn ferrites is $\sim 0.3 \% / \mathrm{K}$ but they are limited to low temperature applications due to their low Curie temperatures $(\sim 450 \mathrm{~K})$.

\section{CONCLUSIONS}

Two characteristic properties that are interesting for soft magnetic applications of HITPERM-type alloys have been studied: permeability and magnetoimpedance. The behavior is in line with previous results on coercivity, and can be explained in terms of domain wall stabilization phenomenon, the difference between the magnetostriction constant of the constituting two phases and grain coarsening. The room temperature softness is enhanced in the alloy with the lowest Co content which exhibits $\mu_{r} \sim 10500$ and a maximum value of MIR $\sim 60 \%$ (at $1 \mathrm{MHz}$ ) for nanocrystalline samples with low crystalline volume fraction. Although the obtained values for the other compositions (39 and 60 at. \% Co) are $\mu_{r} \sim 1000$ and a maximum value of $\mathrm{MIR}<10 \%$ (at $1 \mathrm{MHz}$ ), high temperature applicability is clearly enhanced in these alloys, showing a wide temperature range with approximately constant permeability. This suitability for high temperature applications can be expressed through the temperature coefficient of the permeability $(\sim 0.02 \% / \mathrm{K}$ from room temperature up to $900 \mathrm{~K}$ for the alloy with 39 at. \% Co), 1 order of magnitude lower than those found in FINEMET and Mn ferrites and extended over a much wider temperature range than in these materials.

\section{ACKNOWLEDGMENTS}

This work was supported by the Spanish Government and EU FEDER (Project No. MAT 2001-3175), the PAI of the Junta de Andalucía, the Hispano-Hungarian Bilateral Cooperation Program (Grant No. TÈT E-2/2001) and the Hungarian Scientific Research Fund (OTKA) through Grant No. T-30753. J.S.B. acknowledges a research fellowship of the DGES.

${ }^{1}$ Y. Yoshizawa, S. Oguma, and K. Yamauchi, J. Appl. Phys. 64, 6044 (1988).

${ }^{2}$ K. Suzuki, N. Kataoka, A. Inoue, A. Makino, and T. Masumoto, Mater. Trans., JIM 31, 743 (1990).

${ }^{3}$ M. E. McHenry, M. A. Willard, and D. E. Laughlin, Prog. Mater. Sci. 44, 291 (1999).

${ }^{4}$ A. Hernando, M. Vázquez, T. Kulik, and C. Prados, Phys. Rev. B 51, 3581 (1995)

${ }^{5}$ M. A. Willard, D. E. Laughlin, M. E. McHenry, D. Thoma, K. Sickafus, J. O. Cross, and V. G. Harris, J. Appl. Phys. 84, 6773 (1998).

${ }^{6}$ J. S. Blázquez, V. Franco, A. Conde, M. R. J. Gibbs, H. A. Davies, and Z. C. Wang, J. Magn. Magn. Mater. 250, 260 (2002).

${ }^{7}$ S. He, K. He, B. Shen, H. Zhang, S. Zhang, and H. Guo, J. Appl. Phys. 86, 6301 (1999).

${ }^{8}$ M. A. Willard, M. Q. Huang, D. E. Laughlin, M. E. McHenry, J. O. Cross, and V. G. Harris, J. Appl. Phys. 85, 4421 (1999). 
${ }^{9}$ M. A. Willard, D. E. Laughlin, and M. E. McHenry, J. Appl. Phys. 87, 7091 (2000).

${ }^{10}$ T. Kemény, D. Kaptás, L. F. Kiss, J. Balogh, L. Bujdosó, J. Gubicza, T. Ungár, and I. Vincze, Appl. Phys. Lett. 76, 2110 (2000).

${ }^{11}$ R. S. Beach and A. E. Berkowitz, Appl. Phys. Lett. 64, 3652 (1994).

${ }^{12}$ L. V. Panina and K. Mohri, Appl. Phys. Lett. 65, 1189 (1994).

${ }^{13}$ M. Vázquez, J. Magn. Magn. Mater. 226-230, 693 (2001).

${ }^{14}$ R. Valenzuela, J. J. Freijo, A. Salcedo, M. Vázquez, and A. Hernando, J. Appl. Phys. 81, 4301 (1997).

${ }^{15}$ M. Vázquez, M. Knobel, M. L. Sánchez, R. Valenzuela, and A. P. Zhukov, Sens. Actuators A 59, 20 (1997).

${ }^{16}$ M. Hauser, L. Kraus, and P. Ripka, IEEE Instrumentation and Measurement Magazine (IEEE, Piscataway, NJ, 2001), Vol. 4, p. 28.

${ }^{17}$ Y. Honkura, M. Yamamoto, Y. Kohtani, and K. Mohri, Digest of INTERMAG Europe 2002 Conference, Amsterdam, Netherlands, 2002, CD 10.

${ }^{18}$ H. Lee, Y. K. Kim, T. K. Kim, Y. H. Song, and S. C. Yu, J. Appl. Phys. 85, 5429 (1999).

${ }^{19}$ H. Lee, Y. K. Kim, T. K. Kim, and S. C. Yu, J. Magn. Magn. Mater. 215, 307 (2000)

${ }^{20}$ Y. Y. Dai, Y. H. Liu, S. Q. Xiao, L. Zhang, L. Q. Yue, H. Z. Wu, and Y. Z. Zhang, Phys. Status Solidi A 181, 413 (2000).

${ }^{21}$ H. Q. Guo et al., Mater. Sci. Eng., A 226, 550 (1997).

${ }^{22}$ H. Q. Guo, H. Kronmuller, T. Dragon, C. Chen, and B. G. Shen, J. Appl. Phys. 84, 5673 (1998).

${ }^{23}$ C. Appino, C. Beatrice, M. Coisson, P. Tiberto, and F. Vinai, J. Magn. Magn. Mater. 226, 1476 (2001).

${ }^{24}$ K. S. Kim, S. C. Yu, Y. M. Moon, and H. B. Lee, Phys. Status Solidi A 189, 871 (2002).

${ }^{25}$ H. S. Kwon, H. Lee, K. Kim, S. C. Yu, and Y. K. Kim, J. Alloys Compd. 326, 309 (2001).
${ }^{26}$ J. S. Blázquez, V. Franco, C. F. Conde, and A. Conde, J. Magn. Magn. Mater. 254-255, 460 (2003).

${ }^{27}$ J. S. Blázquez, C. F. Conde, and A. Conde, Appl. Phys. Lett. 79, 2898 (2001).

${ }^{28}$ M. Kersten, Z. Angew. Phys. 8, 313 (1956).

${ }^{29}$ J. S. Blázquez, C. F. Conde, and A. Conde, J. Non-Cryst. Solids 287, 187 (2001).

${ }^{30}$ A. R. Yavari and O. Drbohlav, Mater. Trans., JIM 36, 896 (1995).

${ }^{31}$ J. S. Garitaonandia, D. S. Schmool, and J. M. Barandiarń, Phys. Rev. B 58, 12147 (1998).

${ }^{32}$ J. S. Blázquez, S. Lozano-Pérez, and A. Conde, Philos. Mag. Lett. 82, 409 (2002).

${ }^{33}$ V. V. Shulika and A. P. Potapov, J. Phys. IV 8, Pr2-147 (1998).

${ }^{34}$ B. Hernando, M. L. Sánchez, V. M. Prida, M. Tejedor, and M. Vázquez, J. Appl. Phys. 90, 4783 (2001).

${ }^{35}$ G. V. Kurlyandskaya, J. M. Barandiarán, J. Gutiérrez, D. Garcia, M. Vázquez, and V. O. Vas'kovskiy, J. Appl. Phys. 85, 5438 (1999).

${ }^{36}$ J. Gutiérrez, V. Muto, and P. T. Squire, J. Non-Cryst. Solids 287, 417 (2001).

${ }^{37}$ M. Knobel, J. Schoenmaker, J. P. Sinnecker, R. Sato Turtelli, R. Grössinger, W. Hofstetter, and H. Sassik, Mater. Sci. Eng., A 226-228, 546 (1997).

${ }^{38}$ Y. Zhang, J. S. Blázquez, A. Conde, P. J. Warren, and A. Cerezo, Mater. Sci. Eng. A (accepted).

${ }^{39}$ R. C. Oh'Handley, Modern Magnetic Materials: Principles and Applications (Wiley, New York, 1999).

${ }^{40}$ V. Franco, C. F. Conde, A. Conde, and L. F. Kiss, J. Magn. Magn. Mater. 215-216, 400 (2000). 